\title{
Effects of Batroxobin Injection on Progressive Ischemic Stroke and Serum MMP-2 and MMP-9 Expressions
}

\author{
WeiHai Jiang ${ }^{1, a}$, Wei Sun ${ }^{2, b^{*}}$ and Shu Jing ${ }^{3, c}$ \\ ${ }^{1}$ Department of Orthopedics, Affiliated Hospital, Beihua University, Jilin, Jilin, China \\ ${ }^{2}$ Department of Neurology, Affiliated Hospital, Beihua University, Jilin, Jilin, China \\ ${ }^{3}$ Department of General Surgery, Affiliated Hospital, Beihua University, Jilin, Jilin, China \\ aemail:jiangwehai139@sina.com.com, bemail:sunwei2003jlu@sina.com, cemail:yitonglh@126.com
}

Keywords: Batroxobin; Progressive ischemic stroke (PIS); MMP-2; MMP-9

Abstract. Effects of batroxobin on progressive ischemic stroke (PIS) and serum MMP-2 and MMP-9 expressions in patients with PIS were investigated. 160 Hospitalized patients with acute progressive ischemic stroke were randomly divided into a treatment group $(n=80)$ and a control group $(n=80)$. Patients in the treatment group were treated with the conventional therapy combined with batroxobin injection, in which $10 \mathrm{u}, 5 \mathrm{u}$ and $5 \mathrm{u}$ of batroxobin injection were successively administered once every other day, respectively, and patients in the control group were treated with the conventional therapy alone. Therapeutic effects on PIS and patients' NIHSS scores between the two groups were compared. Serum MMP-2 and MMP-9 expressions before and after the treatment were detected with enzyme-linked immunosorbent assay (ELASE). The results showed that the total effective rate of patients in the treatment group was $91.25 \%$, significantly better than that in the control group, which was $78.75 \%$ ( $\mathrm{P}<0.05)$; NIHSS scores after the treatment were significantly lover than those before the treatment in both groups; compared with those before the treatment, MMP-2 and MMP-9 levels were significantly decreased after the treatment $(\mathrm{P}<0.05)$; differences in NIHSS scores and serum MMP-2 and MMP-9 levels between the two groups were significant after the treatment $(\mathrm{P}<0.05)$. Batroxobin injection can be used for the treatment of PIS, with a definite therapeutic effect. It is confirmed that batroxobin injection can significantly reduce the infarct area, promote the recovery of neurological functions, and delay the development of PIS to improve the prognosis of patients with PIS, which may be closely related to its reducing the level of MMP-2 and MMP-9 in the body.

\section{Introduction}

Ischemic stroke is the most common stroke in clinic and progressive ischemic stroke (PIS) is a particular type of stroke with a high disability rate and mortality. Progressive ischemic stroke refers to a state that symptoms of the neurological function impairment aggravate gradually or stepwise within several days or one week after the onset of an acute ischemic stroke. PIS accounts for a sizeable proportion of acute ischemic stroke, about $30-40 \%$ of patients with acute ischemic stroke ${ }^{[1]}$. The role of matrix metalloproteinase family (MMPS) in the pathogenesis of ischemic stroke has attracted scholars' attention. It is confirmed that matrix metalloproteinase-2 and -9 (MMP-2 and -9) are involved in the process of formation and development of atherosclerosis in ischemic stroke, which has become an increasingly widespread concern ${ }^{[2,3]}$. One of the primary therapeutic methods for PIS is the combined therapy of thrombolysis, anti-platelet aggregation, anticoagulation and improvement of microcirculation. However, the thrombolytic therapy is often limited because most of the patients admitted to hospital miss the optimal time window of thrombolytic therapy, so that more attention has paid to the defibrase treatment. Batroxobin is a single component enzyme extracted from the venom of the Brazilian viper, a defibrase. It can selectively act on the bond between arginine and glycine at the end of fibrinogen $A \alpha$ bond to degradate fibrinogen into fibrin monomer, and then the monomers can polymerize to become polymers that can be broken down by batroxobin into fibrin degradation products; in addition, it also presnets effects such as the decomposition of coagulation factor I , inhibition of thrombosis, lowering of blood viscosity, improvement of red blood cell deformability and decrease in the vascular resistance, etc. ${ }^{[4]}$. In this study, an attempt was made to investigate the 
mechanisms of the vascular function disorders, the aggravation of neurological deficit symptoms, and the intervention with batroxobin by measuring serum MMP-2 and -9 contents in patients with PIS.

\section{Patients and methods}

\section{Clinical data}

160 Patients with PIS confirmed by imageological methods and hospitalized from January 2013 to June 2014 at department of neurology in our hospital were chosen as the research subjects. The inclusion criteria: (1) They all were in line with the diagnostic criteria of ischemic cerebrovascular diseases proposed by The Fourth National Cerebrovascular Disease Conference; (2) The patients' ages were $\leq 80$ years old, and the patients' neurologic deficit scores based on US National Institutes of Health stroke scale (NIHSS) were 4-20 points; (3) Those with cerebral hemorrhage, severe complications, mental illness and drug allergy were excluded. There were 102 males and 58 females in the patients chosen, and their ages were $42-80$ years and the average age was $(65.4 \pm 5.2)$ years. All the patients were randomly divided into a treatment group (80 cases) and a control group (80 cases). Before the onset of ischemic stroke, there had been no one with tumor, infection, acute myocardial infarction, abnormal thyroid function, blood diseases, autoimmune diseases, severe liver and kidney dysfunction and other diseases in the patients included. There was no significantly statistical difference in age, gender, infarct location and history between the two groups $(\mathrm{P}>0.05)$.

\section{Reagents}

ELISA kit was purchased from Shanghai Kangzhuo Technology Co. and batroxobin injection was purchased from Beijing Tobishi Pharmaceutical Co., Ltd.

\section{Therapies}

All patients were treated with the conventional therapy. The patients with diabetes, hypertension and coronary heart disease were given the appropriate basic treatment to adjust the blood glucose, control the blood pressure and stabilize the athrosclerotic plaque based on the patients' conditions, and at the same time, some other basic therapies, such as improvement of circulation, anti-platelet aggregation, nerve nutrition and maintenance of internal homeostasis. The patients in the treatment group were intravenously successively given $10 \mathrm{u}, 5 \mathrm{u}$ and $5 \mathrm{u}$ batroxobin injection (Beijing Tobishi Pharmaceutical Co.) every other day, and the course of treatment was five days.

Specimen collection On the first day before and after the treatment, and the 14th day after the treatment, $3 \mathrm{ml}$ of venous fasting blood from each patient were taken respectively in all the patients. The blood specimen stood for one hour, then was centrifuged at 2000r/min for 10min to separate the serum, and finally was kept in a liquid nitrogen container at $-70^{\circ} \mathrm{C}$ for use.

Determination of MMP-2 and -9 MMP-2 and -9 contents were detected with enzyme-linked immunosorbent assay (ELISA). The specific procedures: (1) The frozen specimen was thawed at room temperature and the standard curve of antibody standard concentration was established according to the kit instruction. (2) according to the requirements of instruction, the antibody standard solution diluted multiply and $100 \mu 1$ specimen were added to the corresponding wells of the plates, the wells were sealed closely with adhesive tape, and then they were incubated at room temperature for $120 \mathrm{~min}$; (3) The plates were washed according to the washing method proposed by the kit instruction;

(4) Except the blank well, $100 \mu l$ of biotin-labeled secondary antibody diluted according to the requirements of kit instruction were added to each well, the wells were sealed in the same way described above, and then they were incubated at room temperature for $90 \mathrm{~min}$; (5)The plates were washed according to the washing method proposed by the kit instruction; (6)Except the blank well, $100 \mu l$ of the diluted enzyme conjugate were added to each well, the wells were sealed, and they were incubated at room temperature for $30 \mathrm{~min}$. (7) The plates were washed according to the washing 
method proposed by the kit instruction; (850 $\mu$ l of substrate A and substrate B were added to each well, and they were kept in dark place flexibly for 10 30 min, depending on the color development; (9) $50 \mu \mathrm{l}$ of the stop solution were added to each well, the absorbance was measured immediately with $450 \mathrm{~nm}$ microplate, the standard curve was drawn based on the measured values, and MMP-2 and -9 expression levels of the serum samples were calculated based on the standard curve ${ }^{[4]}$. The quality control was strictly conducted during the whole testing process.

Judgement criteria of the therapeutic effect

(1) Approximate recovery: patients with decreased functional impairment scores from $91 \%$ to $100 \%$

and class 0 of disability degree; (2) Remarkable recovery: those with decreased functional impairment scores from $46 \%$ to $90 \%$ and class 1 to 3 of disability degree; (3) Somewhat recovery: those with decreased functional impairment scores from $18 \%$ to $45 \%$; (4) Unchanged: those with unchanged functional impairment scores or decreased functional impairment scores only $17 \%$ or less. (5) Exacerbation: those with functional impairment scores that increased less than $18 \%{ }^{[5]}$. The total effective rate was calculated according to the following formula.

Total effective rate $(\%)=$ (basic recovery rate + remarkable recovery rate + somewhat recovery rate)/total number of cases $\times 100 \%$.

Statistical analysis

All the measured data were described with $\mathrm{x} \pm \mathrm{s}$ and processed with statistical software SPSS13.0. Differences between the two groups were compared with $t$-Test and $\mathrm{P}<0.05$ was considered statistically significant.

\section{Results}

\section{Comparison in the therapeutic effect between the two groups}

As shown in Table 1, there was no death in the process of treatment in the two groups and no hemorrhagic in the treatment group, and the total effective rate in the treatment group was significantly higher than that in the control group $(\mathrm{P}<0.05)$.

Table 1 Comparison in the therapeutic effect between the two groups (cases)

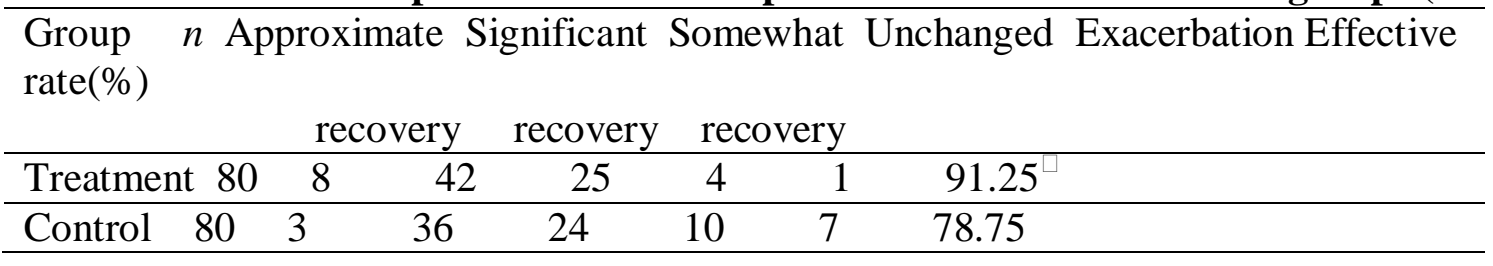

Note : $: \mathrm{P}<0.05$ compared with the control group.

\section{Discussion}

Matrix metalloproteinases (MMPS) is a $\mathrm{Zn}^{2+}$-dependent proteolytic enzyme. With the in-depth study of MMPS in recent years, it was found that the formation, development and prognosis of ischemic stroke are closely related to MMPS. MMP-2 and -9 MMPS are core members of the MMPS family, and secreted by a variety of cells, with the effect to degrade collagen and elastin ${ }^{[5]}$. Under physiological conditions, MMP-2 and MMP -9 exist in a form of inactive zymogen; MMP-2 and MMP -9 may be activated, and then converted to their active forms due to the effect of inflammatory mediators in cerebral ischemia ${ }^{[6]}$, which can destroy the integrity of the vessel wall structure and damage the blood brain barrier, leading to leakage and rupture of the vessel wall due to the rupture of basement membrane, promoting the degradation of the fibrous cap matrix to result in the instability of plaque, and then the thrombosis, finally resulting in the occurrence of ischemic stroke, and even hemorrhagic transformation ${ }^{[7,8]}$. 
Batroxobin injection is a powerful preparation of defibrases. It reduces the concentration of fibrinogen primarily through conversing fibrinogen into soluble fibrinogen, thereby reducing the blood viscosity, improving the blood circulation, lowering the peripheral vascular resistance and increasing the cerebral blood flow to exert its improvement on the blood supply to the brain tissues and inhibition on the thrombosis. Domestic multi-center studies showed that fibrinolytic therapy can effectively improve the prognosis of patients with acute cerebral infarction. The results of the study showed that NIHSS scores of patients in batroxobin-treated group were significantly improved compared with those in the control group, indicating that batroxobin injection has a better therapeutic effect on progressive ischemic stroke; batroxobin injection could effectively down-regulate the expression of MMP-2 and MMP-9, which may play a protective effect on the atherosclerotic plaque on the cerebrovascular endothelial cells by helping to maintain the structure of fibrous cap on the surface of vascular endothelium and keep the vasculopathy at a steady state. It can be concluded that batroxobin may reduce the expression of MMP-2 and MMP-9 to alleviate the cerebral edema and reduce the infarct size, thereby reducing the brain damage. This study may provide an experimental basis for the further study on MMP-2 and MMP-9 inhibitors.

\section{Conclusion}

Batroxobin injection can be used for the treatment of PIS, with a definite therapeutic effect. It is confirmed that batroxobin injection can significantly reduce the infarct area, promote the recovery of neurological functions, and delay the development of PIS, to improve the prognosis of patients with PIS, which may be closely related to its reducing the level of MMP-2 and MMP-9 in the body.

\section{Acknowledgements}

The research work was supported by National Natural Science Foundation of China under Grant No. 61065009 and Natural Science Foundation of Qinghai Provincial under Grant No. 2011-z-756.

\section{References}

[1] Wang Baojun, Liu Bing. Clinical observation on therapeutic effects of batroxobin on acute cerebral infarction[J]. Guide of China Medicine, 2013; 11(4): 455-56.

[2] Lu Zheng, Huo Chunni. Therapeutic effects of edaravone combined batroxobin on aged patients with acute cerebral infarction [J]. Hebei Medical Journal, 2012; 34 (11): 1640-42.

[3] Cao Libo. Analysis on FIB test results in the treatment of patients with acute cerebral infarction with batroxiobin [J]. China Practical Medical, 2011; 6(8): 198-99.

[4] Li Shize, Ding Jinjing, Shi Zhe. Effects of edaravone combined with Xingnaojing injection on serum NSE, S-100 $\beta$ and MMP-9 levels in patients with acute cerebral infarction [J]. Chinese Journal of Gerontology, 2013; 33(2): 273-75.

[5]Wang Tongxin, Zhao Zhuling, Wang Shanjun, et al. Correlation of MMP-2 and MMP-9 expressions in the tissue surrounding hematoma with the cerebral edema of rats with cerebral hemorrhage [J]. Chinese Journal of Gerontology, 2013; 33(4): 885-86.

[6] Zhu Jialei, Deng Xiahang, Gao Li, et al. Effect of resveratrol on NF- $\kappa$ B-induced upregulation of MMP-9 function following cerebral ischemia [J]. Chinese Journal of Gerontology, 2013; 33(5): 1073-75.

[7] Rosenberg GA, Cunningham LA, Wallace J, et al. Immunohistochemistry of matrix metalloproteinases in reperfusion injury to rat brain [J]. Brain Res, 2001, 893:104-12.

[8] Asahi M, Wang X, Mori T, et al. Effects of matrix metallopro-teinase-9 gene knock-out on the proteolysis of blood-brain barrier and white matter components after cerebral ischemia $[\mathrm{J}]$. $\mathrm{J}$

Neurosci, 2001, 21(19): 7724-32. 